\title{
Correction to: Random graph asymptotics on high-dimensional tori Il: volume, diameter and mixing time
}

\author{
Markus Heydenreich ${ }^{1} \cdot$ Remco van der Hofstad ${ }^{2}$ \\ Published online: 9 August 2019 \\ (c) The Author(s) 2019
}

\section{Correction to: Probab. Theory Relat. Fields (2011) 149:397-415 https://doi.org/10.1007/s00440-009-0258-y}

\begin{abstract}
In [3, Theorem 1.2], we claim that the maximal cluster for critical percolation on the high-dimensional torus is non-concentrated. This proof contains an error. In this note, we replace this statement by a conditional statement instead.
\end{abstract}

\section{Correction of [3, Theorem 1.2]}

Recall from [3] that $\mathcal{C}_{(i)}$ denotes the $i$ th largest cluster for percolation on the $d$ dimensional torus $\mathbb{T}_{r, d}$, so that $\mathcal{C}_{(1)}=\mathcal{C}_{\text {max }}$ is the largest component and $\left|\mathcal{C}_{(2)}\right| \leq\left|\mathcal{C}_{(1)}\right|$ is the size of the second largest component, etc. Then, the statement of [3, Theorem 1.2] should be replaced by the following (shortened) statement:

Theorem 1.2 (Random graph asymptotics of the ordered cluster sizes) Fix $d>6$ and $L$ sufficiently large in the spread-out case, or d sufficiently large for nearest-neighbor percolation. For every $m=1,2, \ldots$ there exist constants $b_{1}, \ldots, b_{m}>0$, such that for all $\omega \geq 1, r \geq 1$, and all $i=1, \ldots, m$,

The original article can be found online at https://doi.org/10.1007/s00440-009-0258-y.

Remco van der Hofstad

r.w.v.d.hofstad@tue.nl

Markus Heydenreich

MO.Heydenreich@few.vu.nl; m.heydenreich@1mu.de

1 Department of Mathematics, Vrije Universiteit Amsterdam, De Boelelaan 1081a, 1081 HV Amsterdam, The Netherlands

2 Department of Mathematics and Computer Science, Eindhoven University of Technology, P.O. Box 513, 5600 MB Eindhoven, The Netherlands 


$$
\mathbb{P}_{\mathbb{T}, p_{c}\left(\mathbb{Z}^{d}\right)}\left(\omega^{-1} V^{2 / 3} \leq\left|\mathcal{C}_{(i)}\right| \leq \omega V^{2 / 3}\right) \geq 1-\frac{b_{i}}{\omega}
$$

Consequently, the expected cluster sizes satisfy $\mathbb{E}_{\mathbb{T}, p_{c}\left(\mathbb{Z}^{d}\right)}\left|\mathcal{C}_{(i)}\right| \geq b_{i}^{\prime} V^{2 / 3}$ for certain constants $b_{i}^{\prime}>0$.

In [3, Theorem 1.2], an additional non-concentration result was claimed for $V^{-2 / 3}\left|\mathcal{C}_{\max }\right|$. The proof of this result is incorrect. Below we will explain why, and replace this statement by a conditional version. Unfortunately, we are not able to prove the required condition.

\section{Last paragraph of discussion in [3, Section 1.3]}

In the last paragraph of [3, Section 1.3], we discuss the non-concentration of $V^{-2 / 3}\left|\mathcal{C}_{\max }\right|$, a feature that is highly indicative of the critical behavior. This paragraph needs to be removed.

\section{Corrections to the proof of Theorem 1.2}

The proof of [3, Theorem 1.2] still applies, except for [3, Proposition 3.1], where the non-concentration of $\left|\mathcal{C}_{\max }\right| V^{-2 / 3}$ is proved. This statement can be replaced by the following conditional statement:

Proposition $3.1\left(\left|\mathcal{C}_{\max }\right| V^{-2 / 3}\right.$ is not concentrated) Under the conditions of $[3$, Theorem 1.1], and assuming that there exists $\omega>6^{2 / 3}$ such that

$$
\liminf _{V \rightarrow \infty} V^{1 / 3} \mathbb{P}_{\mathbb{T}, p_{c}\left(\mathbb{Z}^{d}\right)}\left(|\mathcal{C}|>\omega V^{2 / 3}\right)>0,
$$

the random sequence $\left|\mathcal{C}_{\max }\right| V^{-2 / 3}$ is non-concentrated.

The proof of [3, Proposition 3.1] can be followed verbatim, except for the discussion right after [3, (3.19)]. Indeed, [3, (3.19)] reads

$$
\begin{aligned}
\operatorname{Var}_{p_{c}\left(\mathbb{Z}^{d}\right)}\left(Z_{>\omega V^{2 / 3}} V^{-2 / 3}\right) \geq & V^{-1 / 3} \mathbb{P}_{\mathbb{T}, p_{c}\left(\mathbb{Z}^{d}\right)}\left(|\mathcal{C}|>\omega V^{2 / 3}\right) \\
& \times\left[\omega V^{2 / 3}-V \mathbb{P}_{\mathbb{T}, p_{c}\left(\mathbb{Z}^{d}\right)}\left(|\mathcal{C}|>\omega V^{2 / 3}\right)\right] \\
\geq & V^{1 / 3} \mathbb{P}_{\mathbb{T}, p_{c}\left(\mathbb{Z}^{d}\right)}\left(|\mathcal{C}|>\omega V^{2 / 3}\right)\left[\omega-C_{\mathcal{C}} \omega^{-1 / 2}\right],
\end{aligned}
$$

and below it, we claim that this remains uniformly positive for $\omega \geq 1$ sufficiently large, by $[3,(2.4)]$. The problem is that $[3,(2.4)]$ applies only to $\omega$ that are not too large, while to keep the second factor in (3.3) positive, we need to take $\omega>0$ sufficiently large, which we cannot satisfy simultaneously.

An inspection of the proof of the upper bound in [3, (2.4)] (which is originally [1, Theorem 1.3]) shows that $C_{\mathcal{C}}=6$ suffices. Indeed, by [2, Proposition 2.1], $\mathbb{P}_{\mathbb{T}, p_{c}\left(\mathbb{Z}^{d}\right)}(|\mathcal{C}| \geq k) \leq \mathbb{P}_{\mathbb{Z}, p_{c}\left(\mathbb{Z}^{d}\right)}(|\mathcal{C}| \geq k)$. Further, by $[4,(9.2 .6)], \mathbb{P}_{\mathbb{Z}, p_{c}\left(\mathbb{Z}^{d}\right)}(|\mathcal{C}| \geq$ 
$k) \leq \frac{\mathrm{e}}{\mathrm{e}-1} M\left(p_{c}\left(\mathbb{Z}^{d}\right), 1 / k\right)$, while [4, Lemma 9.3] proves that $M\left(p_{c}\left(\mathbb{Z}^{d}\right), \gamma\right) \leq \sqrt{12 \gamma}$. Thus, we need that $\omega>6^{2 / 3}$ to keep the second term in (3.3) strictly positive. For this choice, also [3, (3.19)] is satisfied. As a result, the proof can be repaired when we assume (3.2) for $\omega>6^{2 / 3}$.

Mind that $[3,(2.4)]$ implies (3.2) for $\omega<b_{\mathcal{C}}$ for a positive constant $b_{\mathcal{C}}$ (which is the same as $b_{1}$ in [1, Theorem 1.3]). The actual value of $b_{\mathcal{C}}$ depends of the position of $p_{c}\left(\mathbb{Z}^{d}\right)$ within the critical window of $p_{c}\left(\mathbb{T}_{r, d}\right)$. While [3, Theorem 2.1] guarantees that $p_{c}\left(\mathbb{Z}^{d}\right)$ does lie within the critical window, it gives us no control on the precise position.

We believe that (3.2) is correct, in fact, even for all $\omega>0$. For example, for the Erdôs-Rényi random graph model, which is the corresponding mean-field model, a corresponding statement is true for all $\omega>0$, cf. [5, Lemma 2.2], where even a local limit version of (3.2) is proved. However, we have not been able to show this for percolation on the high-dimensional torus.

Acknowledgements The work of $\mathrm{RvdH}$ is supported by the Netherlands Organisation for Scientific Research (NWO), through VICI Grant 639.033.806 and the Gravitation NETWORKS Grant 024.002.003.

\section{References}

1. Borgs, C., Chayes, J., van der Hofstad, R., Slade, G., Spencer, J.: Random subgraphs of finite graphs. I. The scaling window under the triangle condition. Random Struct. Algorithms 27(2), 137-184 (2005)

2. Heydenreich, M., van der Hofstad, R.: Random graph asymptotics on high-dimensional tori. Commun. Math. Phys. 270(2), 335-358 (2007)

3. Heydenreich, M., van der Hofstad, R.: Random graph asymptotics on high-dimensional tori II: volume, diameter and mixing time. Probab. Theory Relat. Fields 149(3-4), 397-415 (2011)

4. Heydenreich, M., van der Hofstad, R.: Progress in High-Dimensional Percolation and Random Graphs. CRM Short Courses Series. Springer, Cham (2017)

5. van der Hofstad, R., Kager, W., Müller, T.: A local limit theorem for the critical random graph. Electron. Commun. Probab. 14, 122-131 (2009)

Publisher's Note Springer Nature remains neutral with regard to jurisdictional claims in published maps and institutional affiliations. 